\title{
GMR
}

\section{Development and characterization of novel microsatellite markers for Ginkgo biloba using 454 pyrosequencing}

\author{
Q. Zhou ${ }^{1 *}$, X.X. Wang ${ }^{1 *}$, M. Xu ${ }^{1}$, F.L. Cao ${ }^{1}$, F.X. $\mathbf{Y u}^{2}$ and L.A. $\mathbf{X u}^{1}$ \\ ${ }^{1}$ Co-Innovation Center for Sustainable Forestry in Southern China, \\ Nanjing Forestry University, Nanjing, China \\ 2Jiangxi Provincial Key Laboratory of Ornamental Plant Genetic Improvement, \\ Research Institute for Bioresources, Jiangxi Academy of Sciences, Nanchang, \\ China
}

*These authors contributed equally to this study.

Corresponding author: L.A. Xu

E-mail: laxu@njfu.edu.cn

Genet. Mol. Res. 15 (1): gmr.15017385

Received August 6, 2015

Accepted November 13, 2015

Published March 28, 2016

DOI http://dx.doi.org/10.4238/gmr.15017385

ABSTRACT. As a "living fossil" that is used to understand the evolutionary history of seed plants, Ginkgo biloba is a well-known multipurpose tree with edible seeds, medicinal properties, and ornamental value, but little is known about its genetic diversity. Microsatellite, or simple sequence repeat (SSR), markers have proven to be powerful tools for genetic studies of plants. In this study, we isolated 30 novel polymorphic microsatellite loci in G. biloba using 454 pyrosequencing. The characteristics of these loci were tested with 48 cultivars. The number of alleles $\left(N_{\mathrm{A}}\right)$ per locus ranged from two to seven. The observed $\left(H_{0}\right)$ and expected $\left(H_{\mathrm{E}}\right)$ heterozygosities ranged from 0.000 to 0.750 and from 0.021 to 0.792 , with an average of 0.326 and 0.443 , respectively. In terms of genetic diversity in the Ginkgo population, $N_{\mathrm{A}}$ was $3.300, N_{\mathrm{E}}$ was 2.090 , I was $0.782, H_{\mathrm{O}}$ was 0.326 , and $H_{\mathrm{E}}$ 
was 0.443. These polymorphic SSRs will be useful for the assessment of population genetic diversity and resource conservation of G. biloba.

Key words: Ginkgo biloba; 454 Pyrosequencing; Microsatellites; Genetic diversity

\section{INTRODUCTION}

Ginkgo biloba, also known as the maidenhair tree, is one of the oldest living tree species on the planet. This tree is considered to be a "living fossil", meaning that it has continued to survive even after major extinction events. Thus, understanding the origin and phylogeography of $G$. biloba will help to elucidate the evolutionary history of seed plants, and the climate and geological changes that have occurred over time in the Northern Hemisphere. Its natural habitat is restricted to small areas of China (Shen et al., 2005; Gong et al., 2008; Tang et al., 2012), but this species is now widely cultivated around the world due to its edible seeds, medicinal properties, and ornamental value. Despite considerable field surveys and extensive fossil records for the genus Ginkgo (Zhou and Zheng, 2003; Zhou, 2009; Zhou et al., 2012), little is known about population genetics, molecular ecology, and genetic resources of this species. Microsatellites, or simple sequence repeats (SSRs), consist of tandem arrays of short nucleotide motifs, which are randomly dispersed throughout eukaryotic genomes. SSR markers have distinguishing features such as reproducibility, abundant polymorphism, and co-dominant inheritance. These make SSRs powerful high-resolution tools for the study of population genetics, molecular ecology, and marker-assisted selection (MAS) in plants.

Although a few SSR markers are available, genomic resources for G. biloba remain limited (Yan et al., 2006; Li et al., 2009; Xu et al., 2015). Owing to the urgent need for germplasm conservation in this species, and its important role in phylogenetic studies, the generation of transcriptome data will contribute to the conservation of these trees and to genetic research in gymnosperms. In this study, 30 novel microsatellite markers were isolated and characterized from G. biloba based on 454 pyrosequencing.

\section{MATERIAL AND METHODS}

\section{Sample collection and DNA extraction}

Young leaves of 48 Ginkgo cultivars from the major planting areas of the species were collected from the Pizhou Ginkgo Germplasm Garden in Jiangsu Province. These samples were individually ground to powder in liquid nitrogen, and DNA extraction was performed using a DNeasy Plant Mini Kit (Qiagen).

\section{SSR development and genotyping}

Total RNA was extracted from the female cones and leaves of a single individual using an RNeasy Plant Mini Kit (Qiagen). Methods for cDNA library construction and 454 pyrosequencing have been previously described (Chen et al., 2011). Subsequently, SSRs were detected using the MIcroSAtellite (MISA) program search module. The parameters were set to detect perfect di-, tri-, tetra-, penta-, and hexanucleotide motifs, with a minimum of nine, six, five, five, and four repeats, 
respectively. SSR primer pairs were designed using the Primer Premier5.0 software.

PCRs were performed under the following conditions: an initial denaturation at $94^{\circ} \mathrm{C}$ for 5 min, followed by 30 cycles of $30 \mathrm{~s}$ at $94^{\circ} \mathrm{C}, 30 \mathrm{~s}$ at the locus-specific annealing temperature, and $40 \mathrm{~s}$ at $72^{\circ} \mathrm{C}$, followed by a final extension of $1 \mathrm{~min}$ at $72^{\circ} \mathrm{C}$. A typical $10-\mu \mathrm{L}$ reaction contained: $1 \mathrm{X}$ buffer, $2.5 \mathrm{mM} \mathrm{MgCl}_{2}, 0.2 \mathrm{mM}$ each dNTPs, $0.25 \mu \mathrm{M}$ each primer, $0.25 \mathrm{U}$ Taq DNA polymerase (Takara), and 25 ng genomic DNA. Polymorphic PCR products were analyzed on $8 \%$ silver-stained polyacrylamide gels.

\section{Data analysis}

Genetic diversity indices, including the number of alleles at each locus $\left(N_{\mathrm{A}}\right)$, the number of effective alleles per locus $\left(N_{E}\right)$, Shannon's information index $(I)$, observed heterozygosity $\left(H_{\mathrm{O}}\right)$, and expected heterozygosity $\left(H_{\mathrm{E}}\right)$ were estimated using GenAIEx version 6.5 (Peakall and Smouse, 2012).

\section{RESULTS AND DISCUSSION}

Two cDNA libraries were constructed from female cones and leaves of a single individual, and sequenced on the Roche 454 GS-FLX platform. In total, 251,636 raw reads with an average length of $345 \mathrm{bp}$ were generated from female cones, and 223,261 raw reads with an average length of $346 \mathrm{bp}$ were generated from leaves. After adaptor removal and assembly by Newbler version 2.3 (Roche Applied Science, USA) using default parameters, 19,128 contigs and 24,940 singletons were generated from female cones, and 14,671 contigs and 15,108 singletons were generated from leaves.

The MISA program was used for SSR detection. Among 44,068 sequences derived from female clones, a total of 605 perfect SSRs were identified, including 274 dinucleotide repeats, 206 trinucleotide repeats, 42 tetranucleotide repeats, 27 pentanucleotide repeats, and 56 hexanucleotide repeats. Of 19,779 sequences from leaves, a total of 365 perfect SSRs were identified, including 179 dinucleotide repeats, 116 trinucleotide repeats, 24 tetranucleotide repeats, 6 pentanucleotide repeats, and 40 hexanucleotide repeats (Table 1).

Table 1. Frequency, type, and distribution of simple sequence repeats (SSRs) in Ginkgo biloba.

\begin{tabular}{l|c|c}
\hline & Clones & Leaves \\
\hline No. of sequences examined & 44,068 & 19,779 \\
\hline No. of SSR-containing sequences & 562 & 336 \\
\hline Total number of perfect SSRs & 605 & 365 \\
\hline No. of dinucleotide motifs & 274 & 179 \\
\hline No. of trinucleotide motifs & 206 & 116 \\
\hline No. of tetranucleotide motifs & 42 & 24 \\
\hline No. of pentanucleotide motifs & 27 & 6 \\
\hline No. of hexanucleotide motifs & 56 & 40 \\
\hline No. of compound SSRs & 23 & 22 \\
\hline
\end{tabular}

A total of 556 non-redundant SSR-containing sequences were utilized for primer design. Of 556 SSRs, 176 primer pairs produced polymorphic products in six Ginkgo individuals. The characteristics of 30 SSR loci were then investigated using Ginkgo cultivars. Among the 30 SSR loci 
amplified, 15 were dinucleotide repeats; seven were trinucleotide repeats; two were tetracleotide repeats; and six were hexanucleotide repeats (Table 2). In total, 99 alleles were detected in all 48 G. biloba individuals. The $N_{\mathrm{A}}$ per locus ranged from two to seven. The $H_{\mathrm{O}}$ and $H_{\mathrm{E}}$ ranged from 0.000 to 0.750 and from 0.021 to 0.792 , with an average of 0.326 and 0.443 , respectively (Table 1 ).

Table 2. Characterization of 30 polymorphic microsatellite markers for Ginkgo biloba, including locus name, repeat motif, primer sequences, expected size of alleles $(S)$, number of alleles $\left(N_{A}\right)$, number of effective alleles $\left(N_{\mathrm{E}}\right)$, observed heterozygosity $\left(H_{\mathrm{O}}\right)$, and expected heterozygosity $\left(H_{\mathrm{E}}\right)$.

\begin{tabular}{|c|c|c|c|c|c|c|c|c|}
\hline & Repeat motif & $S(\mathrm{bp})$ & Forward primer $\left(5^{\prime}\right.$ to 3 & (5' to $\left.3^{\prime}\right)$ & $N_{\mathrm{A}}$ & $N_{\mathrm{E}}$ & $\mathrm{Ho}_{\mathrm{O}}$ & $H_{E}$ \\
\hline GbeSSR16 & $(\mathrm{AG})_{14}$ & 236 & GATCCATTTTCTGGTTCT & GTGTGATTTCTTCCTTGT & 4 & 2.3 & 0.313 & 0.558 \\
\hline GbeSSR25 & $(\mathrm{AT})_{11}$ & 288 & CATTACAGCGACTGAAACA & AGAGTGGGCTTAGCTTGAT & 4 & 2.6 & 0.396 & 0.612 \\
\hline GbeSSR32 & $(\mathrm{TA})_{11}$ & 196 & TTCGCTGTAGCATTTGTG & GCAGGTTGTATTTCGGAG & 3 & 2.3 & 0.458 & 0.571 \\
\hline GbeSSR78 & $(\mathrm{CTT})_{7}$ & 314 & CTGAAGACGGAAACCACCT & GCCGAAAACAAGAACAATG & 3 & 1.7 & 0.021 & 0.401 \\
\hline GbeSSR79 & $(\mathrm{AT})_{10}$ & 192 & GGCAATCAGAATACCTATC & TAAAGCCTACATCACATCC & 2 & 1.2 & 0.208 & 0.187 \\
\hline GbeSSR91 & $(\mathrm{AAC})_{8}$ & 297 & ACCCTCCCAGAAAAAGTC & AGGTTGGCAATGTTAGCA & 5 & 3.1 & 0.458 & 0.675 \\
\hline GbeSSR94 & $($ TTTC)6 & 201 & AGTCCCATTGACCTTTTG & GATCGGCATATTTACTATTC & 2 & 1.1 & 0.125 & 0.117 \\
\hline GbeSSR120 & $(\mathrm{CA})_{11}$ & 234 & AAGTCATAAGCGACAGTG & CCGTCTTTCAGATCAATA & 7 & 4.8 & 0.750 & 0.792 \\
\hline GbeSSR137 & $(\mathrm{AGC})_{6}$ & 384 & ATTCTCCCCACTCTCATC & CTGTAACTTCTGCACTAGC & 2 & 1.1 & 0.083 & 0.080 \\
\hline GbeSSR150 & $(\mathrm{TC})_{9}$ & 160 & ACATAGTGAGAGTCAGCAT & AGAGATACAATACAGAAAGG & 3 & 2.4 & 0.271 & 0.587 \\
\hline GbeSSR156 & $(\mathrm{GA})_{18}$ & 281 & CTGTAACTAACTAATGAGA & GGTAGTGTATTTTTGAAC & 2 & 1.0 & 0.042 & 0.041 \\
\hline GbeSSR202 & $(\mathrm{AG})_{14}$ & 267 & CCCTTGTTTCTCCATAAT & TGCTCATATAGGTGCTCT & 5 & 1.8 & 0.333 & 0.430 \\
\hline GbeSSR215 & $(\mathrm{AAGCAG})_{5}$ & 157 & AGGAACTGATGATGACGAT & СACTTTCACTTTAATAACGG & 4 & 2.1 & 0.292 & 0.534 \\
\hline GbeSSR237 & $(\mathrm{TTG})_{7}$ & 252 & ATCTTCAАСССССТСААСТ & CACGATCACATGCAAATATAC & 4 & 2.5 & 0.354 & 0.597 \\
\hline GbeSSR245 & $(\mathrm{TCCCAC})_{6}$ & 320 & TCCGAGACCGTCGCAATA & TGGTTCCCTCCCAATGAT & 3 & 1.2 & 0.000 & 0.192 \\
\hline GbeSSR367 & $(\text { CATGGA })_{5}$ & 170 & ACTGGGTGGAATACTGAT & CAACTACAAAGTGGAAGA & 3 & 1.9 & 0.292 & 0.467 \\
\hline GbeSSR383 & $(\text { AT })_{14}$ & 299 & CCGATGTAAGCAGGTCAG & GCATTCGTGTCATTGTTG & 4 & 2.6 & 0.458 & 0.615 \\
\hline GbeSSR400 & $(\text { GTAT) })_{5}$ & 244 & GTCTTATATGTGCTTCAGC & CAACTCGAACTATATTACCT & 2 & 1.9 & 0.313 & 0.482 \\
\hline GbeSSR430 & $(\text { TAGGAT })_{5}$ & 325 & TATCGTGTCGTGGAACCG & CCGAATACAAAGCAGCCT & 2 & 1.5 & 0.396 & 0.342 \\
\hline GbeSSR432 & $(A G G C G G)_{4}$ & 287 & GGAGACAAATAGCGGTAA & CACAAGCGTCATCATTCT & 2 & 1.9 & 0.375 & 0.486 \\
\hline GbeSSR463 & $(\mathrm{TA})_{15}$ & 350 & CAGCAATGGAGACTTCTT & GCCAATACTCTTTAACGG & 4 & 2.8 & 0.417 & 0.644 \\
\hline GbeSSR495 & $(\text { AT })_{15}$ & 357 & GAGAAACATCAAGGAGAGT & ATAATAAGGGCATTGTGAG & 4 & 3.0 & 0.646 & 0.663 \\
\hline GbeSSR496 & $(\text { TTA })_{7}$ & 317 & GATTGTGAAAAGAGAAGG & ATTGTAGATTGCTCCAAC & 5 & 3.5 & 0.750 & 0.717 \\
\hline GbeSSR502 & $(\text { AT })_{10}$ & 188 & GAATAGAAGAGATGTGCG & CATATGTTAGTTTGTGGG & 3 & 2.1 & 0.688 & 0.523 \\
\hline GbeSSR503 & $(\mathrm{TC})_{14}$ & 229 & AACTTATTAGCAATCCTCG & CACACATTTACTGAACCTAT & 2 & 1.3 & 0.188 & 0.234 \\
\hline GbeSSR511 & $(\text { TTC })_{6}$ & 424 & TCCCATTTCACCAGTCTC & CTCGTCCTCTCCATCACT & 3 & 2.4 & 0.438 & 0.578 \\
\hline GbeSSR536 & $(\mathrm{GCA})_{6}$ & 176 & AAACAACCACAACCGCCA & CCTTCGCTCCATTCGTCTC & 2 & 1.2 & 0.063 & 0.135 \\
\hline GbeSSR538 & $(\mathrm{CT})_{11}$ & 103 & AGAGATTTTGCGACAGAGC & GGTAGCAGTTGAACCGTTA & 5 & 2.7 & 0.500 & 0.627 \\
\hline GbeSSR549 & $($ AATGGT) 4 & 274 & ATGGCTGCCCTCAACTTG & TGGACTGCTTGGCCTTAG & 2 & 1.0 & 0.021 & 0.021 \\
\hline GbeSSR550 & $(\mathrm{TG})_{12}$ & 154 & $\begin{array}{l}\text { GTTACAGGCAGATTATACC } \\
\end{array}$ & ATGGCACTAACCACACAG & 3 & 1.6 & 0.125 & 0.389 \\
\hline
\end{tabular}

The assessment of population genetic diversity performed using these 30 novel SSR markers will be helpful for the effective management and sustainable utilization of G. biloba resources. In terms of genetic diversity in the 48 Ginkgo cultivars, $N_{\mathrm{A}}$ was 3.300, $N_{\mathrm{E}}$ was 2.090, I was $0.782, H_{\mathrm{O}}$ was 0.326 , and $H_{\mathrm{E}}$ was 0.443 .

The once diverse Ginkgo group is today represented by a single species, G. biloba, which is now widely cultivated around the world. The novel polymorphic SSR markers developed in this study will be available for studies of conservation genetics for G. biloba.

\section{Conflicts of interest}

The authors declare no conflict of interest. 


\section{ACKNOWLEDGMENTS}

Research supported by the Special Fund for Forest Scientific Research in the Public Welfare (\#201204403-3), the Opening Fund for Jiangxi Provincial Key Laboratory of Ornamental Plant Genetic Improvement (\#2013-KLB-01), and the Priority Academic Program Development of Jiangsu Higher Education Institutions (PAPD).

\section{REFERENCES}

Chen Y, Jiang XM, Zhang L, Zhang XY, et al. (2011). Transcriptome characterization of Camellia Sect. Oleifera based on the 592499 ESTs. Sci. Silvae Sin. 47: 161-163.

Gong W, Zeng Z, Chen YY, Chen C, et al. (2008). Glacial refugia of Ginkgo biloba and human impact on its genetic diversity: evidence from chloroplast DNA. J. Integr. Plant Biol. 50: 368-374.http://dx.doi.org/10.1111/j.1744-7909.2007.00375.x

Li YY, Zang LP and Chen XY (2009). Development of polymorphic microsatellite markers for Ginkgo biloba L. by database mining. Conserv. Genet. Resour. 1: 81-83. http://dx.doi.org/10.1007/s12686-009-9019-6

Peakall R and Smouse PE (2012). GenAIEx 6.5: genetic analysis in Excel. Population genetic software for teaching and research--an update. Bioinformatics 28: 2537-2539.http://dx.doi.org/10.1093/bioinformatics/bts460

Shen L, Chen XY, Zhang X, Li YY, et al. (2005). Genetic variation of Ginkgo biloba L. (Ginkgoaceae) based on cpDNA PCRRFLPs: inference of glacial refugia. Heredity (Edinb) 94: 396-401.http://dx.doi.org/10.1038/sj.hdy.6800616

Tang CQ, Yang Y, Ohsawa M, Yi SR, et al. (2012). Evidence for the persistence of wild Ginkgo biloba (Ginkgoaceae) populations in the Dalou Mountains, southwestern China. Am. J. Bot. 99: 1408-1414.http://dx.doi.org/10.3732/ajb.1200168

Xu M, Xu LA, Cao FL, Zhang HJ, et al. (2015). Development of novel chloroplast microsatellite markers for Ginkgo biloba. Genet. Mol. Res. 14: 7715-7720.http://dx.doi.org/10.4238/2015.July.13.17

Yan XF, Lian CL and Hogetsu T (2006). Development of microsatellite markers in ginkgo (Ginkgo biloba L.). Mol. Ecol. Notes 6: 301-302. http://dx.doi.org/10.1111/j.1471-8286.2006.01134.x

Zhou Z and Zheng S (2003). The missing link in Ginkgo evolution. Nature 423: 821-822.http://dx.doi.org/10.1038/423821a

Zhou ZY (2009). An overview of fossil Ginkgoales. Palaeoworld 18: 1-22. http://dx.doi.org/10.1016/j.palwor.2009.01.001

Zhou ZY, Quan C and Liu YS (2012). Tertiary Ginkgo ovulate organs with associated leaves from North Dakota, U.S.A., and their evolutionary significance. Int. J. Plant Sci. 173: 67-80. http://dx.doi.org/10.1086/662651 